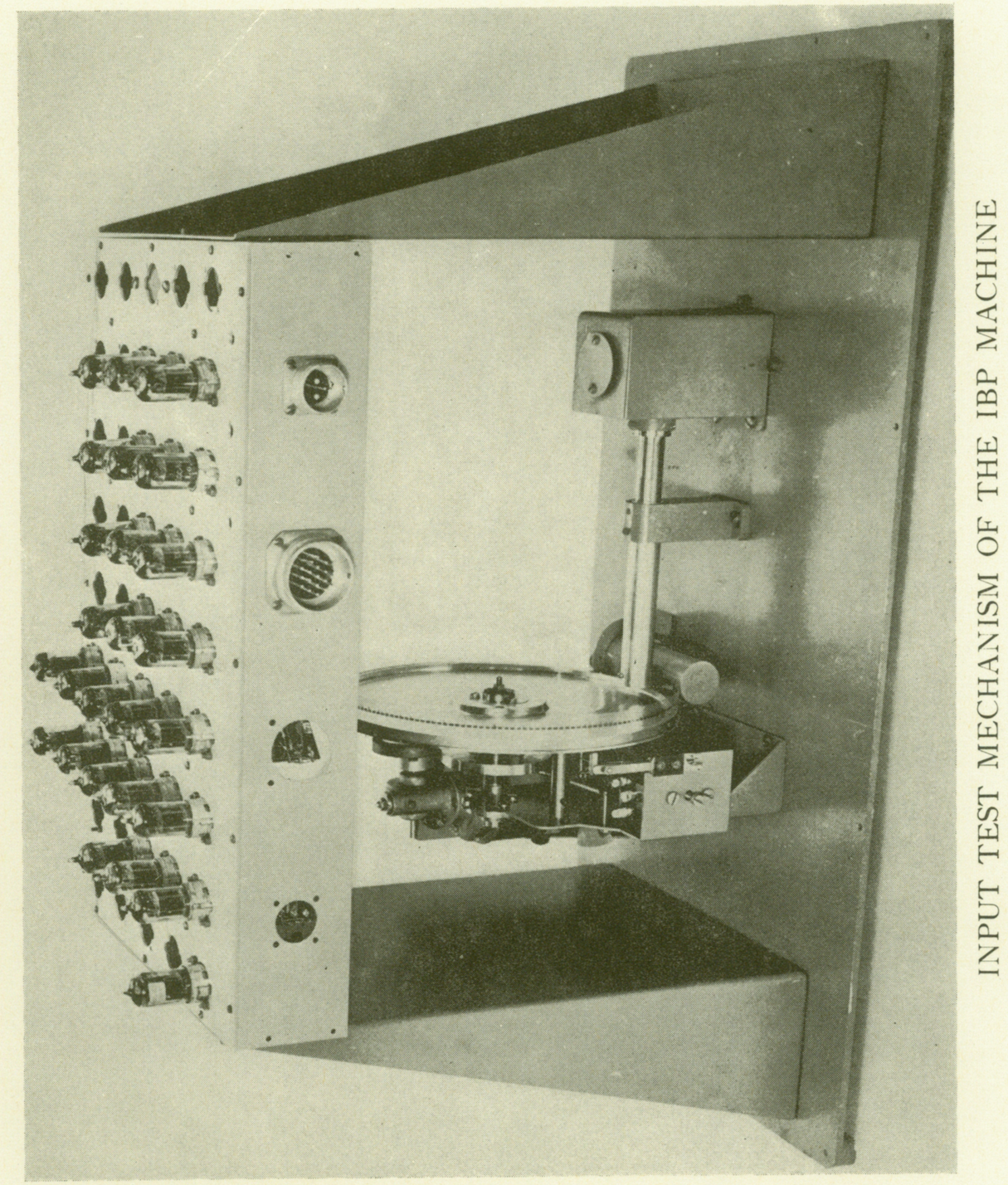




\section{On a Special Case of a Quadrature Formula of Christoffel}

1. This note deals with the numerical evaluation of the integral

$$
\int_{-1}^{1} w(x) f(x) d x
$$

where the special case $a=1$ of the weight function $w(x)=\left(a^{2}+x^{2}\right)^{-1}$ is treated.

Consider quite generally the integral

$$
\int_{\alpha}^{\beta} w(x) f(x) d x
$$

where for the weight function $w(x)$ we assume only that the moments $\mu_{r}=$ $\int_{\alpha}^{\beta} x^{r} w(x) d x$ exist. It is then known ${ }^{1}$ that $n$ properly chosen values of the function, $f\left(x_{\nu}\right), \nu=1,2, \cdots, n$, suffice to give the integral exactly by the sum $\sum_{v=1}^{n} p_{v} f\left(x_{\nu}\right)$, provided $f(x)$ is a polynomial of degree not higher than $2 n-1$. The integration formula thus yields the same accuracy with $n$ ordinates as, e.g., Newton-Cotes formula using $2 n$ equidistant values. The division points $x_{v}\left(\alpha<x_{v}<\beta\right)$ are the zeros of certain polynomials $F_{n}(x)$ of degree $n$, orthogonal with respect to the weight function $w(x)$. The above quadrature formula was first derived by GaUss for the special case $w(x)=1 ; F_{n}(x)$ is then the LEGENDRE polynomial of degree $n$. Similar quadrature formulae of the Gaussian type, as we will call them, have been established for the weight functions

$$
\begin{aligned}
& w(x)=(x-\alpha)^{\gamma}(\beta-x)^{\lambda}, \gamma>-1, \lambda>-1, \\
& w(x)=x^{\lambda} e^{-x}, \alpha=0, \beta=\infty, \lambda>-1, \\
& w(x)=\exp \left(-x^{2}\right), \beta=-\alpha=\infty,
\end{aligned}
$$

where the arguments are the zeros of the JACOBI, LAGUERRE and HERMite polynomials. ${ }^{1}$ As far as the author is aware no quadrature formula associated with the weight function $w(x)=\left(a^{2}+x^{2}\right)^{-1}$ has been derived before. It has, therefore, been considered worth while to treat the case $\beta=-\alpha=1$, for which division points $x_{v}$ and weights $p_{v}$ are supplied for $\nu=2(1) 7$ and $a=1$.

The importance of quadrature formulae of the Gaussian type for purely numerical work is admittedly restricted through the irrationality of the arguments which necessitates cumbersome interpolation. For problems connected with the numerical solution of integral or integro-differential equations, however, the Gaussian formulae have proved to be of great value. Considering, e.g., a FREDHOLM integral equation of the second kind

$$
f(x)=g(x)+\int_{\alpha}^{\beta} w(y) f(y) K(x, y) d y
$$

we have approximately

$$
f(x)=g(x)+\sum_{n=1}^{n} p_{v} f\left(y_{v}\right) K\left(x, y_{v}\right) .
$$


Putting $x=y_{v}$, we can generally solve the system for the unknowns $f\left(y_{v}\right)$, and as soon as these basic function values are known, $f(x)$ can be computed from (3) for any value of the argument $x$. This procedure was first employed for the numerical solution of integral equations by Nyström. ${ }^{2}$ The same procedure of replacing the integral by a weighted sum for numerical solution of integro-differential equations has been utilized by WICK ${ }^{3}$ and CHANDRASEKHAR. ${ }^{4}$

2. The natural starting point for establishing the quadrature formulae is the orthogonal property characteristic of the polynomials $F_{n}(x)$. The following procedure for deriving $F_{n}(x)$ is due to CHRIsTOFFEL. ${ }^{5}$ The polynomial $F_{n}(x)$ is orthogonal with respect to $w(x)$, if

$$
\int_{\alpha}^{\beta} w(x) F_{m}(x) F_{n}(x) d x=0, m \neq n ; m, n=0,1, \ldots
$$

or

$$
\int_{\alpha}^{\beta} x^{k} w(x) F_{n}(x) d x=0, n=1,2, \ldots ; k<n .
$$

Defining the quantities

$$
I_{n}=\int_{\alpha}^{\beta} w(x) F_{n}^{2}(x) d x ; k_{n} I_{n}=\int_{\alpha}^{\beta} x w(x) F_{n}^{2}(x) d x,
$$

the following recurrence formula holds for any three consecutive orthogonal polynomials

$$
F_{n+1}(x)=\left(x-k_{n}\right) F_{n}(x)-I_{n} F_{n-1}(x) / I_{n-1} .
$$

It is here required that the coefficient of $x^{n}$ in the polynomial $F_{n}(x)$ is equal to +1 .

The construction of the polynomials can thus be performed from (7) as soon as we know $F_{0}$ and $F_{1}(x)$.

The weights are given by

$$
p_{r}=\frac{1}{F_{n}^{\prime}\left(x_{r}\right)} \int_{\alpha}^{\beta} w(x) \frac{F_{n}(x)}{x-x_{r}} d x, r=1,2, \ldots, n .
$$

Re-writing this as follows

$$
p_{r}=\frac{-1}{F_{n}^{\prime}\left(x_{r}\right) F_{n+1}\left(x_{r}\right)} \int_{\alpha}^{\beta} w(x) \frac{F_{n+1}(x) F_{n}\left(x_{r}\right)-F_{n+1}\left(x_{r}\right) F_{n}(x)}{x-x_{r}} d x,
$$

and making use of the CHRISTOFFEL-DARBOux identity

$$
\sum_{n=0}^{n} F_{\nu}(x) F_{\nu}(y) / I_{\nu}=\left[F_{n+1}(x) F_{n}(y)-F_{n}(x) F_{n+1}(y)\right] /\left[(x-y) I_{n}\right]
$$

we get

$$
p_{r}=\frac{-I_{n}}{F_{n}^{\prime}\left(x_{r}\right) F_{n+1}\left(x_{r}\right)} \sum_{r=0}^{n} \frac{F_{\nu}\left(x_{r}\right)}{I_{\nu}} \int_{\alpha}^{\beta} w(x) F_{v}(x) d x .
$$


But from (5) it is clear that all integrals with the exception of $\int_{\alpha}^{\beta} w(x) F_{d} d x$ vanish, and consequently the following representation holds. ${ }^{1}$

$$
p_{r}=-I_{n} /\left[F_{n}{ }^{\prime}\left(x_{r}\right) F_{n+1}\left(x_{r}\right)\right] \text {. }
$$

Observing that

$$
F_{n+1}\left(x_{r}\right)=-I_{n} F_{n-1}(x) / I_{n-1}
$$

we obtain the alternative formula

$$
p_{r}=I_{n-1} /\left[F_{n}{ }^{\prime}\left(x_{r}\right) F_{n-1}\left(x_{r}\right)\right] \text {. }
$$

3. In the case to be discussed we have $w(x)=\left(a^{2}+x^{2}\right)^{-1} ; \beta=-\alpha=1$. As the integrand $x w(x) F_{n}{ }^{2}(x)$ is an odd function, $k_{n}$ is zero, and

$$
F_{n}(x)=x F_{n-1}(x)-I_{n-1} F_{n-2}(x) / I_{n-2},
$$

which can be used for constructing $F_{n}(x)$. Christoffel ${ }^{5}$ has communicated the following general expression for $F_{n}(x)$

$$
F_{n}(x)=P_{n}(x)-\sigma_{n} P_{n-2}(x) / \sigma_{n-2},
$$

where $P_{n}(x)$ is the Legendre polynomial of degree $n$, and

$$
\sigma_{n}=\sigma_{n}(a i)=2 W_{n-1}(a i)+2 i P_{n}(a i) \operatorname{arccot} a,
$$

$i$ being the imaginary unit. The polynomial $W_{n-1}(u)$ of degree $n-1$ is expressed by the formula

$$
\begin{aligned}
W_{n-1}(u)=\frac{2 n-1}{1 \cdot n} P_{n-1}(u)+\frac{2 n-5}{3(n-1)} & P_{n-3}(u) \\
& +\frac{2 n-9}{5(n-2)} P_{n-5}(u)+\ldots .
\end{aligned}
$$

For $P_{n}(x)$ and associated functions reference is made to E. W. HoBson. ${ }^{6}$ The quantities $\sigma_{n}(i)$ and $P_{n}(i)$ are listed in Table 1 for $n=0(1) 7$.

TABle 1

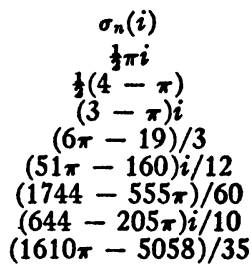

$$
\begin{gathered}
P_{n}(i) \\
1 \\
i \\
-2 \\
-4 i \\
17 / 2 \\
37 i / 2 \\
-41 \\
-92 i
\end{gathered}
$$$$
\begin{gathered}
I_{n} \\
-\frac{1}{3}(4-\pi) \\
8(\pi-3) /(3 \pi) \\
8(19-6 \pi) / 45(4-\pi) \\
8(51 \pi-160) / 1575(\pi-3) \\
8(1744-555 \pi) / 11025(19-6 \pi) \\
512(205 \pi-644) / 121275(51 \pi-160) \\
1024(5058-1610 \pi) / 693693(1744-555 \pi)
\end{gathered}
$$

The division points $-1<x_{v}<+1$ are thus the roots of the equation

$$
F_{n}(x)=P_{n}(x)-\left(\sigma_{n} / \sigma_{n-2}\right) P_{n-2}(x)=0 .
$$

We observe the following properties of $F_{n}(x)$ : The quotient $\sigma_{n} / \sigma_{n-2}$ is always real, the zeros of $F_{n}(x)=0$ are distributed as the zeros of $P_{n}(x)=0$, and 
consequently symmetrical with respect to $x=0$. For $n$ odd, $x=0$ satisfies (19).

Inserting (16) in (6) we obtain an explicit expression of $I_{n}$. As the coefficient of $x^{n}$ differs from +1 we put

$$
I_{n}=c_{n} \int_{-1}^{1} w(x) F_{n}^{2}(x) d x,
$$

where $w(x)=\left(a^{2}+x^{2}\right)^{-1}$. We get

$$
\begin{array}{r}
I_{n} c_{n}^{-1}=\int_{-1}^{1}\left(a^{2}+x^{2}\right)^{-1} P_{n}^{2}(x) d x+\left(\sigma_{n} / \sigma_{n-2}\right)^{2} \int_{-1}^{1}\left(a^{2}+x^{2}\right)^{-1} P_{n-2}{ }^{2}(x) d x \\
-2\left(\sigma_{n} / \sigma_{n-2}\right) \int_{-1}^{1}\left(a^{2}+x^{2}\right)^{-1} P_{n}(x) P_{n-2}(x) d x
\end{array}
$$

The first and last of these integrals can be evaluated as follows:

$$
\int_{-1}^{1}\left(a^{2}+x^{2}\right)^{-1} P_{n}^{2}(x) d x=-i \sigma_{n} P_{n}(a i) / a
$$

and

$$
\int_{-1}^{1}\left(a^{2}+x^{2}\right)^{-1} P_{n}(x) P_{n-2}(x) d x=-i \sigma_{n} P_{n-2}(a i) / a .
$$

Introducing (22) and (23) in (21) we find

$$
I_{n} c_{n}^{-1}=-i\left(\sigma_{n} / \sigma_{n-2}\right)\left[\sigma_{n-2} P_{n}(a i)-\sigma_{n} P_{n-2}(a i)\right] / a,
$$

which also can be written as follows, if we insert (17) in the bracket,

$$
I_{n} c_{n}^{-1}=-2 i\left(\sigma_{n} / \sigma_{n-2}\right)\left[W_{n-3}(a i) P_{n}(a i)-W_{n-1}(a i) P_{n-2}(a i)\right] / a \text {. }
$$

For $c_{n}$ we have the following expression

$$
c_{n}=[n ! /(1 \cdot 3 \ldots(2 n-1))]^{2} .
$$

TABLE 2

\begin{tabular}{clc}
$n$ & \multicolumn{1}{c}{ $\pm x_{v}$} & \multicolumn{1}{c}{$p_{v}$} \\
2 & 0.5227232 & 0.5000000 \\
3 & 0 & 0.5061318 \\
& 0.7438173 & 0.2469341 \\
4 & 0.3151531 & 0.3583405 \\
& 0.8445005 & 0.1416595 \\
5 & 0 & 0.3368260 \\
& 0.5132821 & 0.2404139 \\
& 0.8965229 & 0.09117313 \\
6 & 0.2253363 & 0.2700545 \\
& 0.6410942 & 0.1664560 \\
7 & 0.9264497 & 0.06348956 \\
& 0 & 0.2521628 \\
& 0.3888781 & 0.2065587 \\
& 0.7263167 & 0.1206198 \\
& 0.9451281 & 0.04674011
\end{tabular}


The quantities $I_{n}$ for $a=1$ are given in Table 1 while arguments and weights computed for $n=2(1) 7$ are listed in Table 2 .

A. REIZ

Lund Univ.

Lund, Sweden

${ }^{1}$ G. Szegö, Orthogonal Polynomials, Amer. Math. Soc., Colloquium Publs., v. 23, 1939, chap. III \& XV.

i E. J. NYSTRÖM, "Uber die praktische Auflösung von Integralgleichungen mit Anwendungen auf Randwertaufgaben," Acta Math., v. 54, 1930, p. 185-204. Integral equations defined within an infinite interval have been discussed from the numerical point of view by A. REIZ, "On the numerical solution of certain types of integral equations," Arkiv Mat. Astr. Fysik, v. 29A, no. 29, 1943, 21 p.

3G. C. Wick, "Uber ebene Diffusionsprobleme," Z. Physik, v. 121, 1943, p. 702-718.

4S. CHANDRASEKHAR, "On the radiative equilibrium of a stellar atmosphere, II," Astrophys. Jn., v. 100, 1944, p. 76-86 and following volumes; see also the same author's Radiative Transfer, Oxford Univ. Press, 1950.

5 E. B. CHRISTOFFEL, "Sur une classe particulière de fonctions entières et de fractions continues," Annali di Mat. S.2, v. 8, 1877, p. 1-10.

'E. W. HoBson, The Theory of Spherical and Ellipsoidal Harmonics, Cambridge Univ. Press, 1931, particularly chap. II: $\$ 34$.

\section{New Information Concerning Isaac Wolfram's Life and Calculations}

1. Introductory.-We have already noted certain items ${ }^{1-4}$ regarding Wolfram, an eighteenth century Dutch artillery officer, one of his mathematical tables, and his contacts with LAMBERT. ${ }^{3}$ Hardly anything else is to be found in any mathematical history, periodical, or bibliography-to practically all of which we refer. No one has previously remarked that in two volumes of correspondence of Lambert, edited by one of the Bernoullis (1784, 1785-87), more than 200 pages of material, ${ }^{9}$ including many of Wolfram's letters, tell us much concerning him and his mathematical activities for over 35 years. In what follows my main objects shall be to give some idea of the nature of the new material, and also to include some interesting recently discovered additional facts, supplied by J. H. B. KEMPERMAN, research worker at the Mathematisch Centrum, Amsterdam. Bernoulli refers to the "great calculator Wolfram"9 (v. 5, p. 464), who was notified that the Prussian Academy of Sciences would be glad to receive as a legacy the complete collection of his logarithmic calculations for preservation in its archives.

2. Mr. Kemperman's Report"29 (24 January 1950).- "According to information received from the Royal Military Academy at Breda and the General Public Record Office at The Hague, the following data are certain-

(i) His full name is ISAAC WolfRAM (according to an army list from 1781). He is always indicated by the name J. Wolfram.

(ii) Before 1747 Wolfram was not yet an officer.

(iii) On August 3, 1747 he became "onderlieutenant" (artillery).

(iv) On November 27, 1764 he was promoted to be "ordinairlieutenant."

(v) On September 1, 1779 he became "captain-lieutenant."

(vi) Before 25 August 1788 Wolfram was no longer in the army, for on that date his substitute A. vaN HoEy vaN OOSTEE was sent out (according to the consignment book of the Council of State). In the army-list of 1786 Wolfram's name still appears, but in that of 1789 it is no longer found.

(vii) In 1778 he was stationed at Nijmegen. 